\title{
Reconstrucción inmediata de cuero cabelludo
}

\section{Immediate scalp reconstruction}

Iribarren Brown, 0.*, Ríos Muñoz, P.**, Saavedra Pinto, F.***,

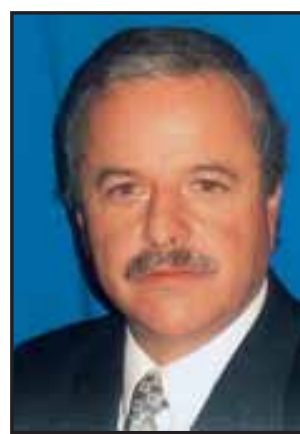

Iribarren Brown, 0 .

Rojas Guzmán, M.***, De Amesti Boza, E.***

\section{Resumen}

Presentamos una serie de casos realizados en el Servicio de Cirugía del Hospital Clínico San Pablo en Coquimbo, Chile, entre Abril de 1998 y Abril de2005. Se trata de un grupo de estudio de 22 pacientes consecutivos con pérdida traumática o quirúrgica del cuero cabelludo, mayor de $3 \mathrm{~cm}$ de diámetro. Se empleó para la reconstrucción colgajo semilunar, en espejo o en remolino vascularizados a partir de las ramas principales del cuero cabelludo. Analizamos en cada caso el tamaño del defecto, pérdida de hueso asociada, tipo de cirugía, resultados de la cobertura y complicaciones.

La edad media de los pacientes fue de 40.6 años con un rango entre 3 y 71 años. El área anatómica comprometida fue parietal en 11 pacientes $(50 \%)$, temporal en $7(31,8 \%)$, occipital en $3(13,6 \%)$ y frontal en 1 caso $(4,5 \%)$. La causa de la pérdida de sustancia fue resección de tumores malignos o benignos en 10 casos, postraumática en 5, postquirúrgica en 4, y por alopecia en 3 . El tamaño medio del defecto fue de $147 \mathrm{~cm}^{2}$ con un rango entre 28 y $615 \mathrm{~cm}^{2}$.

El colgajo empleado fue doble en espejo en 10 casos $(50 \%)$, semilunar de deslizamiento en $9(36,4 \%)$ y en remolino en $3(13,6 \%)$. En 6 pacientes se utilizó un injerto dermo-epidérmico para cerrar un defecto residual del área dadora del colgajo (dos semilunares y dos en espejo). Veintiun pacientes $(45 \%)$ evolucionaron sin incidencias, con cicatrización del colgajo en un plazo máximo de 21 días. Un paciente con pérdida ósea tras dos craneotomías, presentó necrosis parcial distal del colgajo y falleció por neumonía aspirativa.

Comparamos los tres tipos de reparación de acuerdo al tamaño del defecto y a la superficie del mismo. La elección de colgajo doble en espejo, remolino o semilunar, al aplicar la prueba de Kruskal Wallis, no se relacionó con la superficie del defecto, y la elección del colgajo fue atribuible al azar, p > 0.05. La principal razón para escoger uno u otro fue la ubicación del defecto.

En conclusión, la estrategia de reconstrucción del cuero cabelludo mediante colgajos arterializados permite el cierre de grandes defectos en una operación fácil y con cicatrices inaparentes que quedan ocultas bajo el cabello. Es por ello que creemos que estos procedimientos son la mejor alternativa de tratamiento y recomendamos su uso por sus resultados fiables cuando se siguen los principios técnicos del manejo de colgajos.

Palabras clave Cuero cabelludo. Colgajos.

Código numérico 2001-2420
Abstract

We present sucessive cases performed at Surgery Department of Hospital San Pablo, Coquimbo, Chile, between April1998, and April 2005: Twenty two consecutive patients suffering surgical or postinjury scalp defects, greater than 3 $\mathrm{cm}$ of diameter. Semilunar flap, mirror flap, or multiple pinwheel flap arterialized from the main scalp arteries were used for reconstruction. We determinate in every case the large of the defect, asociated bone loss, kind of surgery, end result and complications.

Patients' mean age was 40.6 years with rank between 3 and 71 years old. The involved anatomical area was parietal on 11 patients, $(50 \%)$, temporal in 7 cases $(31.8 \%)$, occipital in 3 cases $(13.6 \%)$ and frontal 1 case, $(4.5 \%)$. The origin of scalp loss was malign or benign tumor resection in 10 patients, post injury 5 cases, secondary to neurosurgical procedures in 4 cases, and alopecia or baldness in 3 cases. The mean large of the defect was $147 \mathrm{~cm}^{2}$ with rank between 28 and $615 \mathrm{cms}^{2}$.

The mirror flap was used in 10 cases $(50 \%)$, the sliding semilunar flap in 9 patients (36.4\%), and the multiple pinwheel flap in 3 patients (13.6\%). An aditional dermoepidermal graft was used on 6 patients to cover a small area of flap donor site (two mirror flaps, and two semilunar flaps). Patients' evolution were without complications in $95 \%$ of cases (21/22). One woman with a temporal bone necrosis after two craniotomies suffered a distal flap necrosis, and finally died due aspirative pneumonia

The three kind of flap were compared according the defect length and defect area. The Kruskal Wallis test shows that the mean length and mean area of three different flap are statistically similars. The choice of one flap or another is no related with the area of the defect, $p>0.05$. The main reason for choosing the flap was the site defect.

Conclutions: the immediate scalp reconstruction strategie by arterialized flaps allows to close big defects, in one step, by a relatively simple technique with hidden scars under hair. We think this procedure is the best choice for immediate scalp recostruction, and we recommend its usage because this technique has reliable outcome when the surgical team follows the technical principles of flaps handle.

Key words Scalp. Flaps.

Código numérico 2001-2420 
Introducción

La pérdida del cuero cabelludo (CC) es tan antigua como el hombre; en Suecia se encontró un cráneo con cuero cabelludo avulsionado correspondiente al año $2180 \mathrm{AC}$. El general romano Orosius (101 A/C) enseñaba a sus soldados estacionados en Italia a que arrancaran el cuero cabelludo de sus enemigos como trofeo de guerra. Entre los pueblos precolombinos de América estaba asentado el concepto de arrancar el cuero cabelludo de los enemigos como signo de castigo y humillación (1).

La primera referencia detallada de tratamiento de una avulsión de CC se hizo en 1870, en la Sociedad Médica de Nebraska: un empleado de Union Pacific sufrió el arrancamiento del cuero cabelludo a manos de un indio. El paciente fue tratado con apósitos saturados con aceite de oliva. Tras supurar profusamente durante tres meses se obtuvo la cicatrización de la calota denudada. A su vez, una comunicación del Nashville Journal of Medicine de 1855 describe a un cirujano francés de apellido Vance, quién entre 1777 y 1791 realizaba en Tennesse múltiples orificios con una lezna de zapatero en el cráneo denudado hasta alcanzar el diploe. Con este procedimiento se producía la cicatrización y cobertura del cráneo en dos años (1).

En la época contemporanea, la pérdida parcial o total del CC es una condición quirúrgica inhabitual. $\mathrm{Su}$ baja incidencia, probablemente ha influido en un escaso interés de los cirujanos para enfrentar su tratamiento. Pérdidas menores de $3 \mathrm{~cm}$. pueden cerrarse a tensión por aproximación de los bordes. Pérdidas mayores generan un desafío, ya que la falta de distensión del CC no permite su cierre primario. Hasta 1970 la mayor parte del tiempo empleado para sanar una pérdida de $\mathrm{CC}$ se destinaba a esperar que creciera tejido de granulación sobre el periostio denudado. Pero había importante controversia respecto a qué hacer una vez conseguido el tejido granulatorio. La recomendación más frecuente era aplicar injertos sobre el mismo mientras existiera epicraneo vital, sorteando así la falta de distensibilidad de la galea aponeurótica que impide la aproximación de los bordes de la herida cuando la pérdida es de más de $2 \mathrm{~cm}$ (2). En los últimos 20 años, los nuevos recursos de la Cirugía Plástica han mejorado esta reconstrucción, acortando el número de operaciones, el tiempo de recuperación y el resultado estético final tras una reconstrucción de cuero cabelludo.

El presente artículo describe la experiencia de nuestro equipo en reconstrucción de CC, e identifica una estrategia de reconstrucción apropiada a partir de un protocolo diseñado según los principios de cierre de CC descritos por Oishi en 1995 (3).
Material y método

El estudio se realiza en el Servicio de Cirugía del Hospital Clínico San Pablo, en Coquimbo, Chile entre Abril 1998 - Abril 2005.

El grupo de estudio está compuesto por 22 pacientes consecutivos con defectos por pérdida traumática o quirúrgica de cuero cabelludo mayores de $3 \mathrm{~cm}$. de diámetro. Durante el período de estudio se identificaron 27 pacientes (5 fueron tratados con expansores (4) y no se incluyen en este estudio).

Analizamos en cada caso el tamaño del defecto, la pérdida de hueso asociada, el tipo de cirugía, los resultados del intento de cobertura y las complicaciones.

Criterios de inclusión: pacientes adultos con pérdida traumática o con lesiones benignas o malignas de $\mathrm{CC}$ que significaron una pérdida del mismo igual o
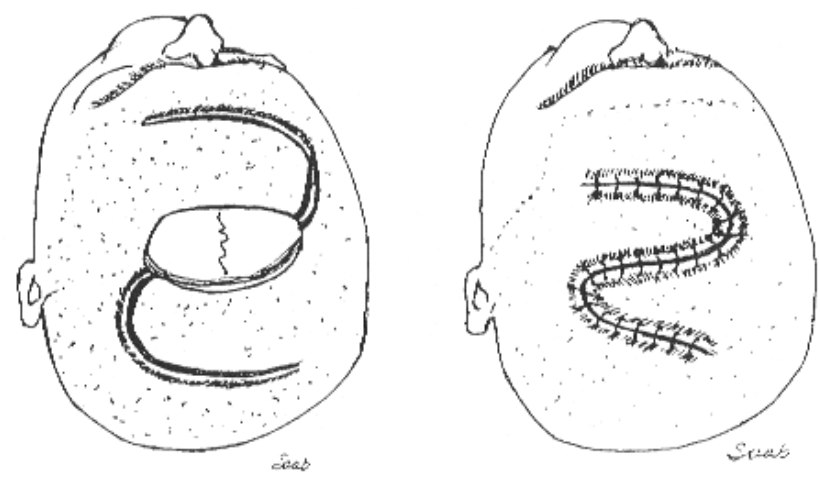

Figura 1: Diseño y cierre del colgajo en espejo.
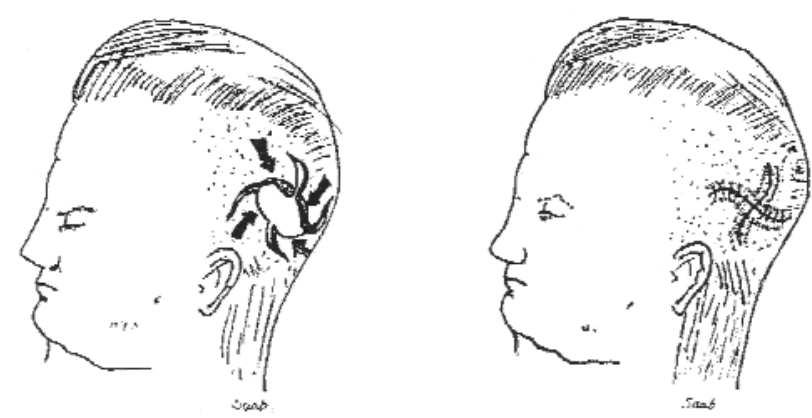

Figura 2: Diseño y cierre del colgajo en remolino.
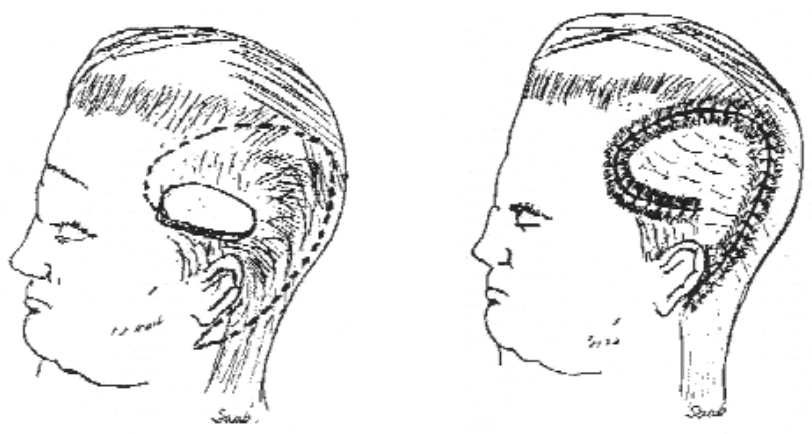

Figura 3: Diseño y cierre del colgajo en media luna. 


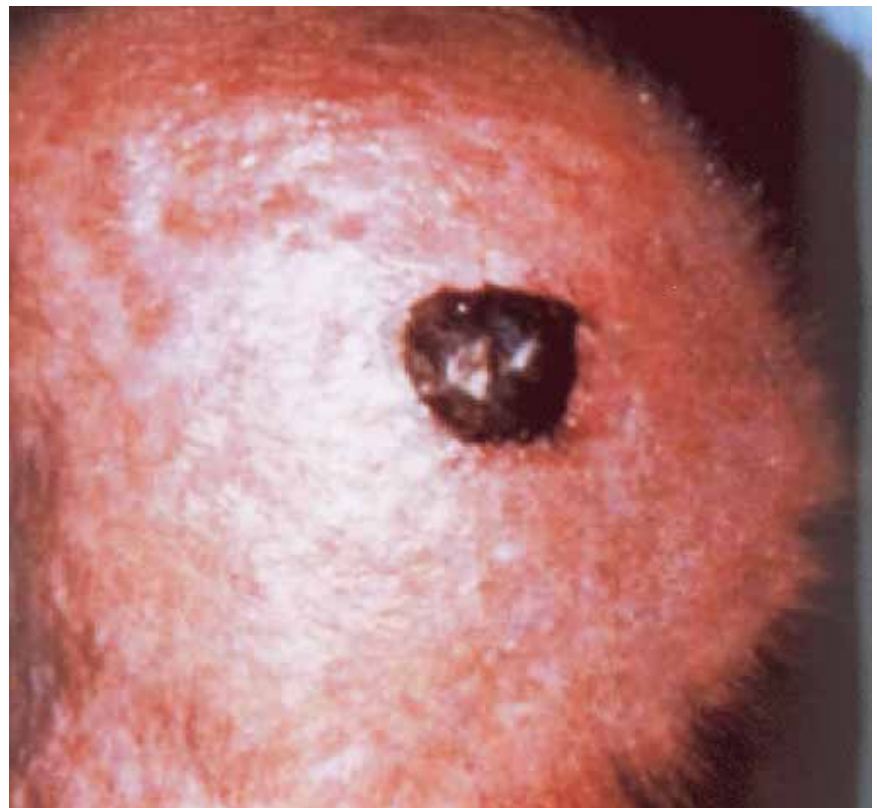

Figura 4 a: Varón de 58 años, preoperatorio melanoma nodular parietal de $2 \mathrm{~cm}$ diámetro.

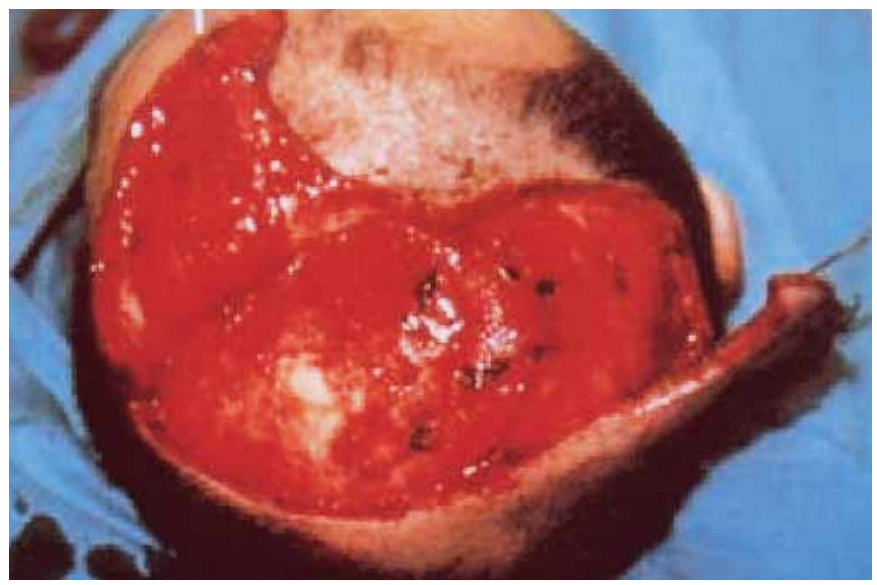

Figura 4 b: Área de resección de melanoma de $6 \mathrm{~cm}$ diámetro y colgajos en espejo preparados; bases de los colgajos en arteria temporal superficial y occipital contralateral.

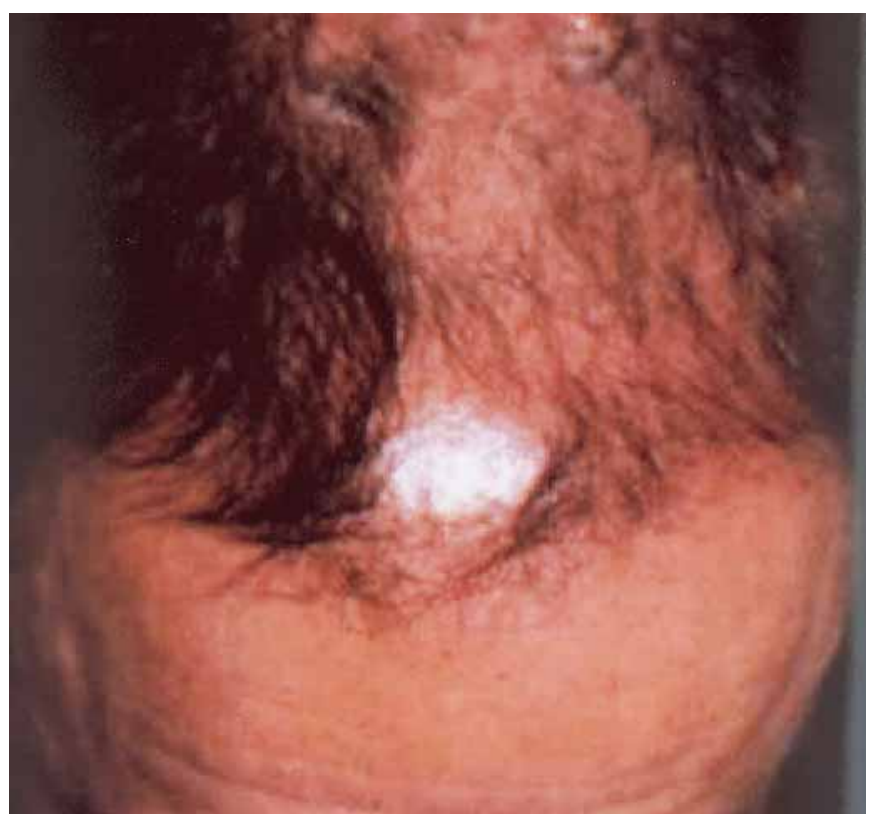

Figura 4 c: Postoperatorio al mes de evolución.
Tabla I. Reconstrucción cuero cabelludo Ubicación del defecto

UBICACIÓN FRECUENCIA PORCENTAJE

$\begin{array}{lcc}\text { PARIETAL } & 11 & 50.0 \\ \text { TEMPORAL } & 7 & 31.8 \\ \text { OCCIPITAL } & 3 & 13.6 \\ \text { FRONTAL } & 1 & 4.5 \\ \text { TOTAL } & \mathbf{2 2} & \mathbf{1 0 0}\end{array}$

mayor de $3 \mathrm{~cm}$ de diámetro. En niños se consideró una pérdida mayor de $2 \mathrm{~cm}$. Los pacientes fueron integrados en un protocolo de reconstrucción inmediata mediante colgajos arterializados a partir de la descripción de la irrigación del cuero cabelludo hecha por Reed y Argenta (2). Todos los pacientes con pérdida de origen traumático fueron evaluados mediante examen físico y radiografías para descartar una lesión asociada de cráneo, cerebromeníngea o cervical, y después operados bajo anestesia general. En los pacientes con pérdida de calota secundaria a operaciones neuroquirúrgicas previas, ésta no fue reparada, postergando su reconstrucción hasta cuatro meses más tarde de acuerdo a principios tradicionales (3). De rutina, la hemostasia de vasos mayores de $2 \mathrm{~mm}$ se realizó con ligaduras de calibre 3/0 absorbibles $\left(\right.$ Vicryl ${ }^{\circledR}$ ) y la hemostasia menor se hizo con electrocoagulación bipolar para reducir el traumatismo por calor del tejido periférico. El cierre de las heridas se realizó en un plano de galea con Vicryl 3/0 y la piel se cerró con grapas.

Para medir la superficie de los defectos circulares se utilizó la fórmula $S=\pi \times r^{2}$ ( $r=$ radio del defecto; $\mathrm{S}=$ superficie), y para los defectos ovales se consideró la fórmula $\mathrm{S}=\mathrm{a} \times \mathrm{b} \times \pi(\mathrm{a}=$ distancia del centro al foco; $\mathrm{b}=$ distancia del centro hasta la parte superior o inferior de la elipse) (5).

Los colgajos utilizados fueron seleccionados por el primer autor a partir de la experiencia clínica previa por su sencillez de diseño, versatilidad y seguridad de manejo. Estos fueron: el colgajo doble en espejo (2) (Fig. 1), el colgajo en remolino (6) (Fig. 2) y el colgajo en media luna, $(3,7)$, (Fig 3$)$.

Como base de datos se utilizó el programa Excel Microsoft. Para el análisis estadístico se escogió la prueba no paramétrica de Kruskal Wallis para medias múltiples del programa S.P.S.S versión 2003. Para validar los resultados se cotejó con la prueba de Mann Withney.

\section{Resultados}

En el período estudiado de 7 años se identificaron 22 pacientes con lesiones de CC en los que se planeó 
Tabla II. Reconstrucción cuero cabelludo. Demografía

\begin{tabular}{|c|c|c|c|c|c|c|c|c|}
\hline caso & $\begin{array}{l}\text { edad } \\
\text { años }\end{array}$ & ubicación & origen & $\begin{array}{l}\text { estado } \\
\text { preoperat }\end{array}$ & $\begin{array}{c}\text { longitud } \\
\mathrm{cm} .\end{array}$ & $\begin{array}{l}\text { superficie } \\
\mathrm{cm}^{2}\end{array}$ & cierre & $\begin{array}{l}\text { injerto } \\
\text { adicional }\end{array}$ \\
\hline 1 & 22 & temporal & cirugía & infectado & 6 & 113,04 & doble & no \\
\hline 2 & 18 & occipital & tu. benigno & sano & 4 & 50,24 & doble & no \\
\hline 3 & 40 & parietal & alopecia & sano & 5 & 78,5 & remolino & no \\
\hline 4 & 32 & parietal & alopecia & sano & 3 & 28,26 & remolino & no \\
\hline 5 & 28 & parietal & alopecia & sano & 3 & 28,26 & remolino & no \\
\hline 6 & 31 & occipital & tu. benigno & sano & 7 & 153,86 & doble & sí \\
\hline 7 & 49 & parietal & traumática & sano & 10 & 314 & doble & sí \\
\hline 8 & 33 & frontal & traumática & infectado & 13 & 530,66 & semilunar & sí \\
\hline 9 & 58 & parietal & melanoma & sano & 6 & 113,04 & doble & no \\
\hline 10 & 15 & temporal & tu. benigno & sano & 10 & 314 & doble & no \\
\hline 11 & 67 & temporal & cirugía & infectado & 14 & 615,44 & doble & sí \\
\hline 12 & 32 & parietal & carcinoma & sano & 5 & 78,5 & semilunar & no \\
\hline 13 & 62 & temporal & cirugía & infectado & 3 & 28,26 & semilunar & no \\
\hline 14 & 3 & parietal & cirugía & infectado & 1 & 3,14 & semilunar & no \\
\hline 15 & 43 & parietal & carcinoma & sano & 4 & 50,24 & doble & no \\
\hline 16 & 47 & parietal & carcinoma & sano & 4 & 50,24 & doble & no \\
\hline 17 & 68 & parietal & carcinoma & sano & 3 & 28,26 & semilunar & no \\
\hline 18 & 70 & occipital & carcinoma & sano & 3 & 28,26 & semilunar & no \\
\hline 19 & 71 & temporal & carcinoma & sano & 3 & 28,26 & semilunar & no \\
\hline 20 & 26 & parietal & traumático & sano & 7 & 153,86 & doble & no \\
\hline 21 & 46 & temporal & traumático & infectado & 8 & 200,96 & semilunar & sí \\
\hline 22 & 44 & temporal & traumático & infectado & 9 & 254,34 & semilunar & sí \\
\hline
\end{tabular}

Tabla III. Colgajo utilizado para cierre del defecto

COLGAJO

FRECUENCIA PORCENTAJE

DOBLE EN ESPEJO
REMOLINO
SEMILUNAR
TOTAL

$\begin{array}{cc}10 & 50 \\ 3 & 13.6 \\ 9 & 36.4 \\ 22 & 100\end{array}$

reconstrucción en un tiempo, 11 varones y 11 mujeres. La edad media de los pacientes fue de 40.6 años, con un rango entre 3 y 71 años. El $50 \%$ de los pacientes tenían una edad entre 27 y 53 años. El área anatómica más frecuentemente comprometida fue la parietal (11 pacientes, $50 \%$ ). Otras áreas tratadas fueron la temporal en 7 casos $(31.8 \%)$, occipital en 3 casos $(13.6 \%)$ y frontal 1 caso $(4.5 \%)$ (Tabla I). En los pacientes con pérdidas traumáticas, hubo 4 casos con más de una región anatómica comprometida (18\%). La causa de la pérdida traumática fue, en todos los casos, accidente con vehículos motorizados.

En 7 pacientes (31.8 \%) había infección local en el momento de la operación. En 4 pacientes había además pérdida de calota y exposición de meninge de tamaño variable, secundaria a procedimientos neuroquirúrgicos repetidos con resultado de necrosis isquémica del hueso u osteomielitis. La longitud (medida en el eje mayor del defecto) fluctuó entre $2.5 \mathrm{~cm}$. (niño de 3 años) y $12.5 \mathrm{~cm}$., con una media de $6 \mathrm{~cm}$. La superficie media del defecto en los adultos fue de
$147,4 \mathrm{~cm} .^{2}$, con un rango entre 28,6 y $615,4 \mathrm{~cm}^{2}$ (Tabla II).

El colgajo empleado para el cierre fue: doble en espejo en 10 casos (50\%) (Fig. 4, 5), semilunar de deslizamiento en 9 pacientes $(36.4 \%$ ) (Fig. 6, 7) y en remolino en 3 pacientes, (13.6\%) (Tabla III) (Fig. 8). En 4 pacientes se utilizó un injerto dermo-epidérmico para cerrar un defecto residual del área dadora del colgajo (dos semilunares y dos en espejo). El $85 \%$ de los pacientes evolucionó sin incidentes y con cicatrización en un plazo máximo de 21 días. Un paciente con exposición ósea y meníngea secundaria a dos craneotomías, presentó necrosis parcial distal del colgajo y falleció por neumonía aspirativa secundaria.

Se compararon los tres tipos de reparación de acuerdo a la media de longitud del defecto y a la superficie del mismo. El uso de colgajo doble en espejo, remolino o semilunar al aplicar la prueba de Mann Withney muestra que la elección de uno u otro colgajo no se relaciona con la superficie de los defectos ( $\mathrm{p}>0.05)$. Al aplicar la prueba de Kruskal Wallis para medias múltiples, comprobamos que la elección del colgajo era atribuible al azar, $(\mathrm{p}>0.05)$, lo que implica que la elección del colgajo no se relaciona con la superficie a reparar.

\section{Discusión}

La pérdida de cuero cabelludo es una patología quirurgica infrecuente. La base de datos de la Biblioteca 


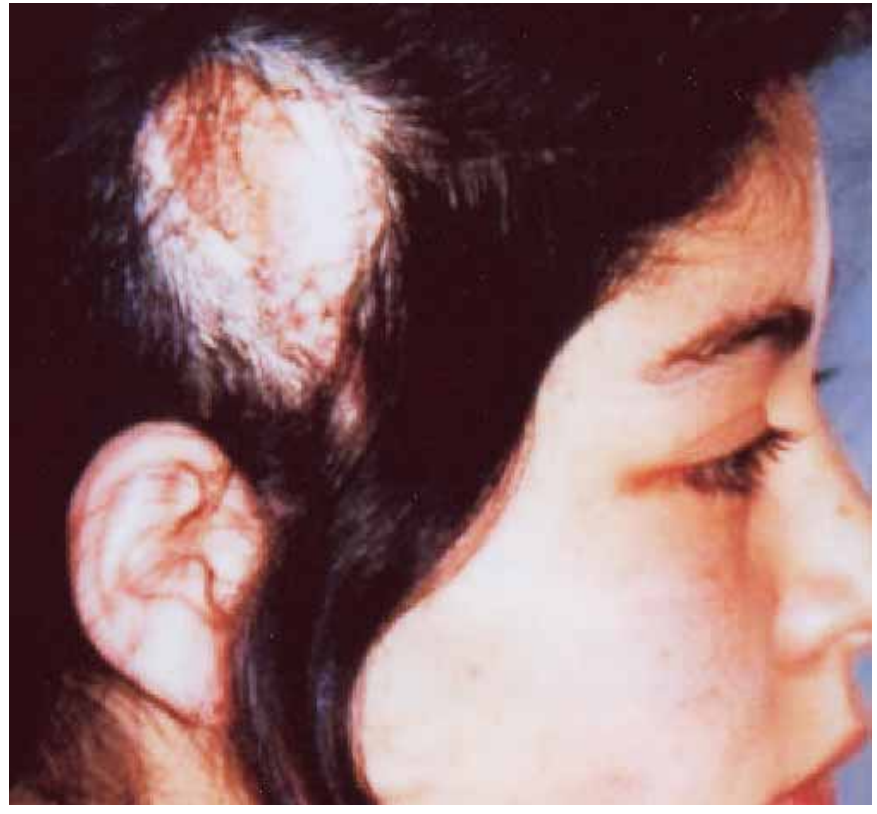

Figura 5 a: Mujer de 15 años, con tumor verrucoso y alopecia temporal de $10 \mathrm{~cm}$ diámetro.

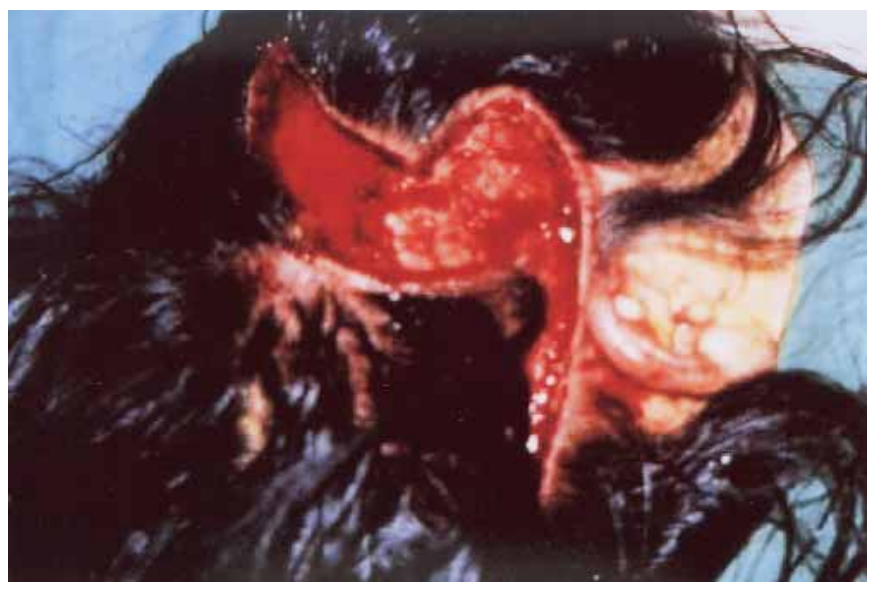

Figura 5 b: Resección de alopecia y preparación de colgajos en espejo; bases en arteria temporal superficial y auricular posterior.

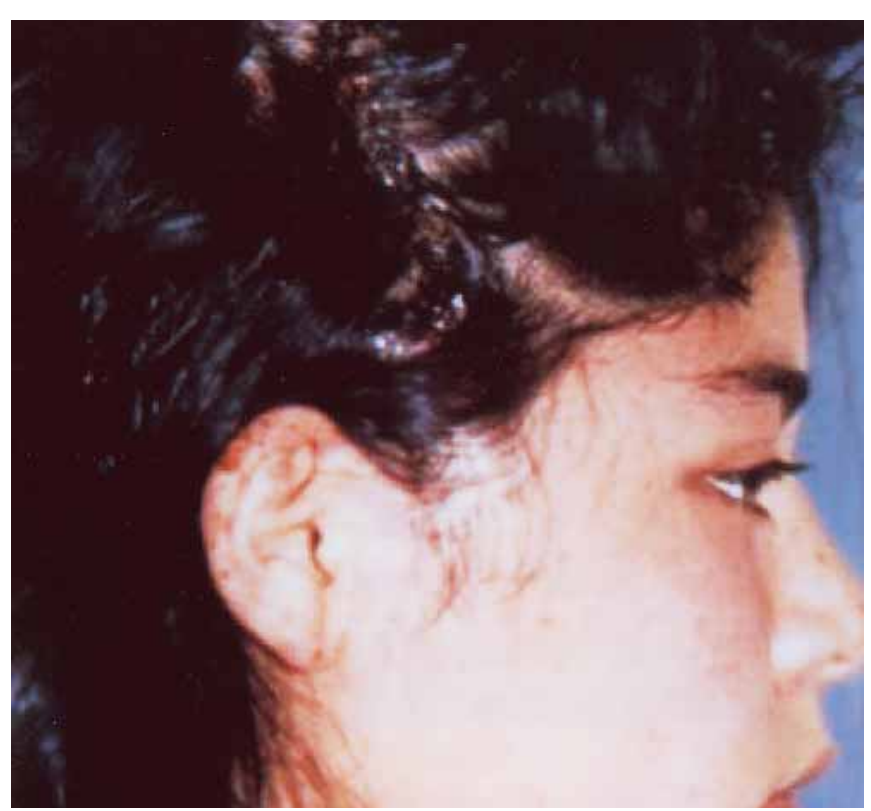

Figura 5 c: Postoperatorio a las 3 semanas, con grapas de cierre in situ.
Nacional de Medicina de EE.UU presenta 12 publicaciones relacionadas con reconstrucción de CC entre 2004 y 2005 en las revistas indexadas ISCI y de esa cantidad, 2 se refieren a pérdidas traumáticas (8). Las causas más frecuentes de pérdida parcial de CC son quemaduras de espesor total, heridas por desgaste, heridas contusas con pérdidas de tejido y resecciones por tumores malignos $(2,9-12)$.

Las pérdidas menores de $3 \mathrm{~cm}$., en los adultos, pueden cerrarse de forma primaria aproximando los bordes, tras la liberación subgaleal a partir de los bordes de la herida. En pérdidas mayores, el método tradicional más simple de cierre es la aplicación de un injerto dermoepidérmico. Una vez que éste ha cicatrizado completamente, la piel injertada puede ser resecada y el defecto se cierra con un colgajo de deslizamiento o con el uso de expansores tisulares $(3,4)$. El uso de colgajos de CC otorga la ventaja de cierre en una sola operación, con relativa facilidad y con cicatrices que quedan ocultas bajo el cabello (13); por esas razones estos procedimientos se han constituido en la mejor alternativa de tratamiento $(14,15)$.

Existen diversos diseños que satisfacen el objetivo; nosotros hemos preferido el semilunar, el doble en espejo y el remolino; todos tienen la dificultad común de que se trata de un tejido inelástico, por ello, la configuración del colgajo debe evaluarse cuidadosamente para evitar la necesidad de cerrar la zona dadora con injertos dermoepidérmicos. Al diseñar el primero de ellos, el borde del colgajo que representa el eje de rotación del mismo debe ser equivalente a cinco largos del defecto, para evitar tensión excesiva de las suturas (16). Para reducir su longitud y aumentar su superficie, utilizamos incisiones múltiples en paralelo de la galea, separadas $1 \mathrm{~cm}$. entre ellas, disminuyendo la resistencia a la distensión del CC y tornándolo más "elástico". Como este procedimiento puede poner en riesgo la vitalidad del colgajo, no hacemos incisiones paralelas en lesiones traumáticas, en las que generalmente el cuero $\mathrm{CC}$ dador del colgajo se encuentra en condiciones inestables de vitalidad. En esos casos el defecto de cierre en el area dadora lo cerramos con injertos dermoepidérmico. Es nuestro colgajo de elección en reconstrucciones postraumáticas.

La alternativa de elección en la unión parieto-occipital ha sido el colgajo en remolino que moviliza los tejidos periféricos en 360 grados, con mínima disección y con distribución homogénea de la tensión sobre las cuatro líneas de sutura radiadas (6), dejando cicatrices inaparentes (fig 5).

La tercera opción es el colgajo doble en oposición, (Fig. 6). Cuando se usa para cubrir la nuca, este colgajo debe diseñarse de modo que sus bases se ubi- 


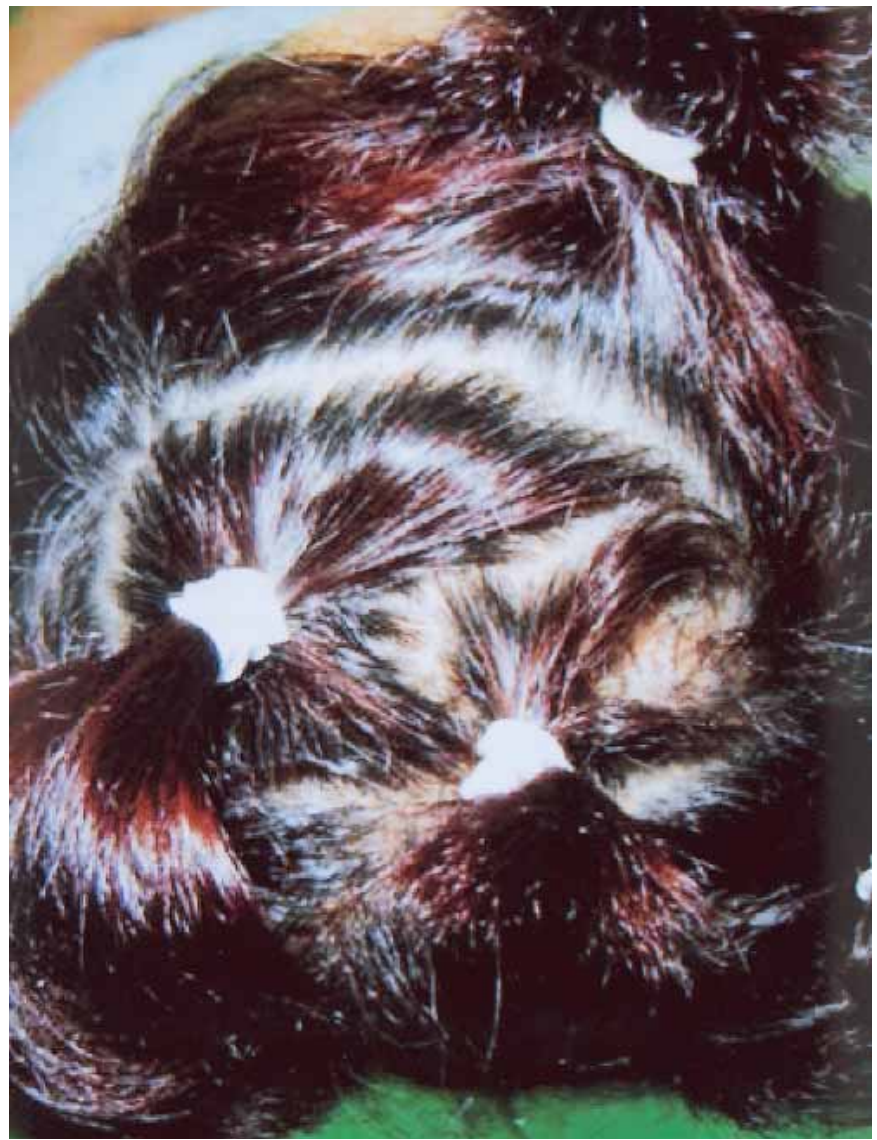

Figura 6 a: Mujer de 32 años con carcinoma adenoquístico parietal derecho.

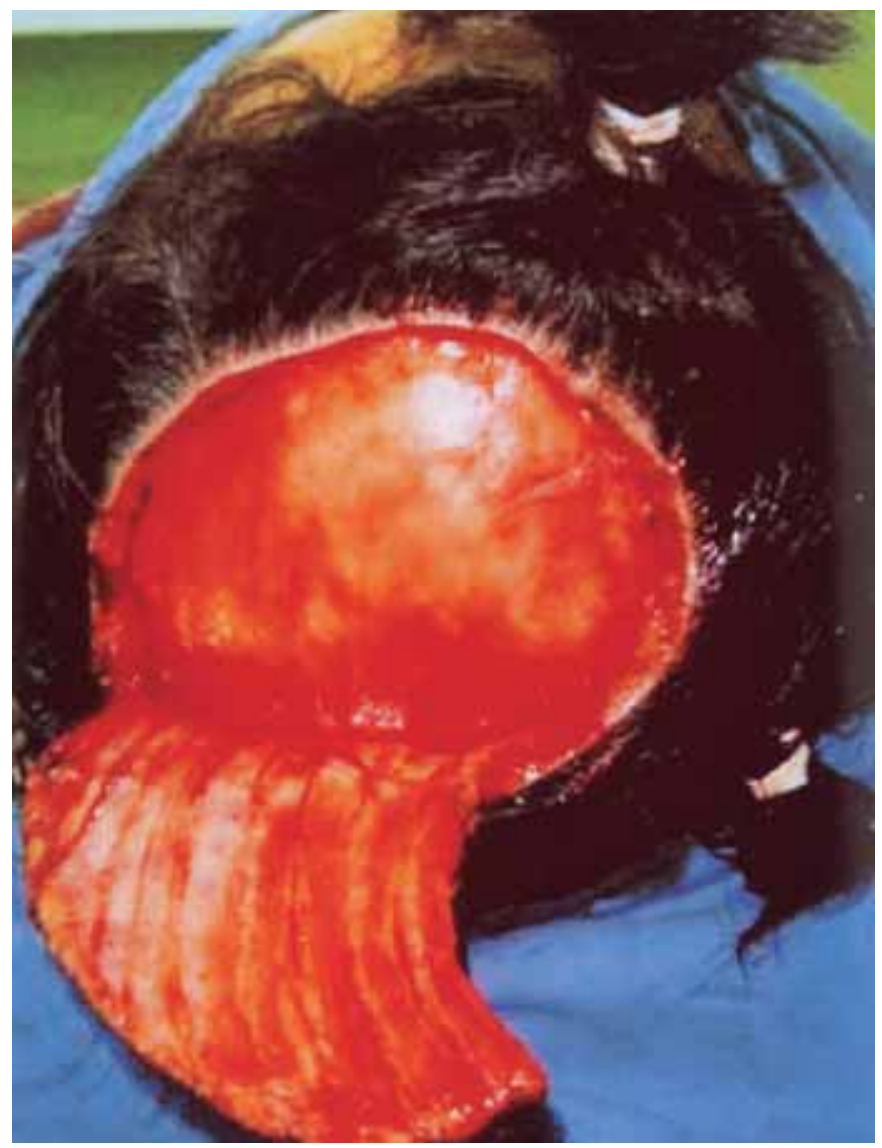

Figura $6 \mathrm{c}$ : Colgajo semilunar parieto-occipital, con incisiones paralelas de galea para aumentar su elasticidad y extensión; base en arteria occipital izquierda.

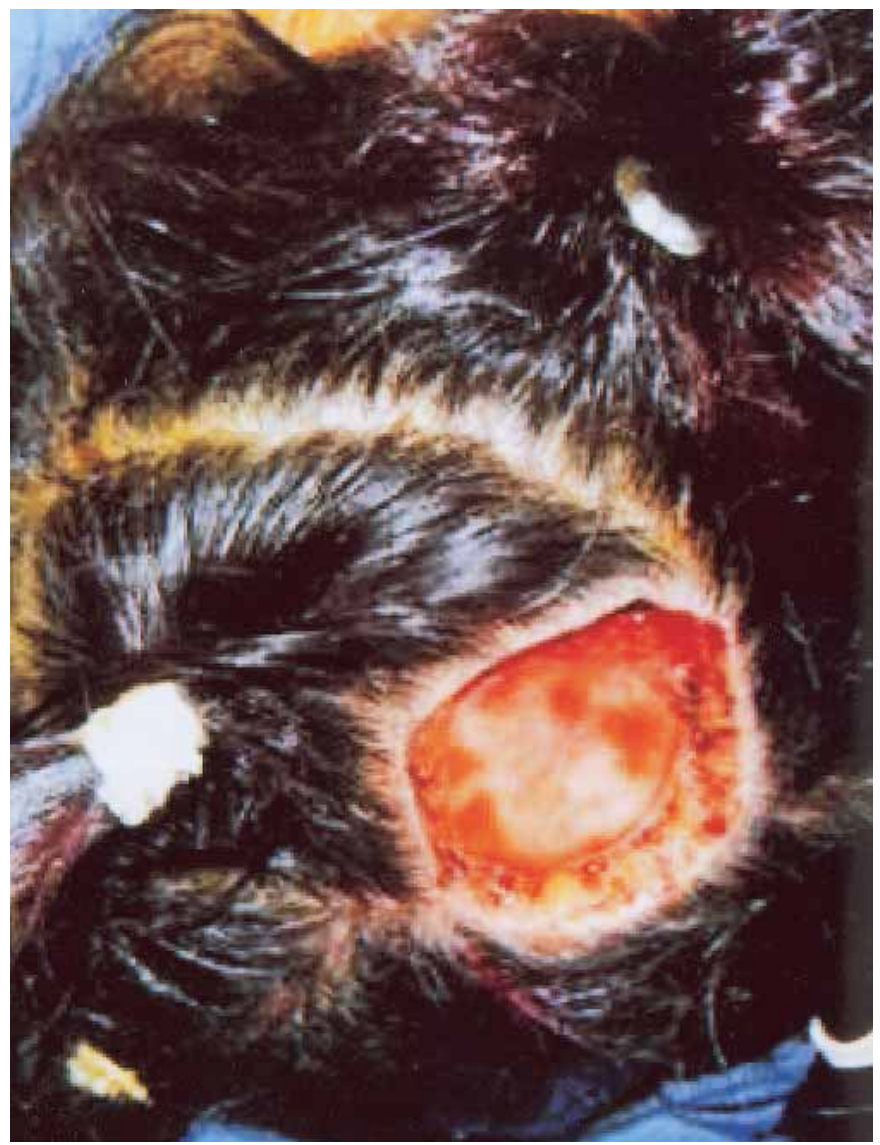

Figura 6 b: Resección de cuero cabelludo, galea y periostio de $5 \mathrm{~cm}$. de diámetro.

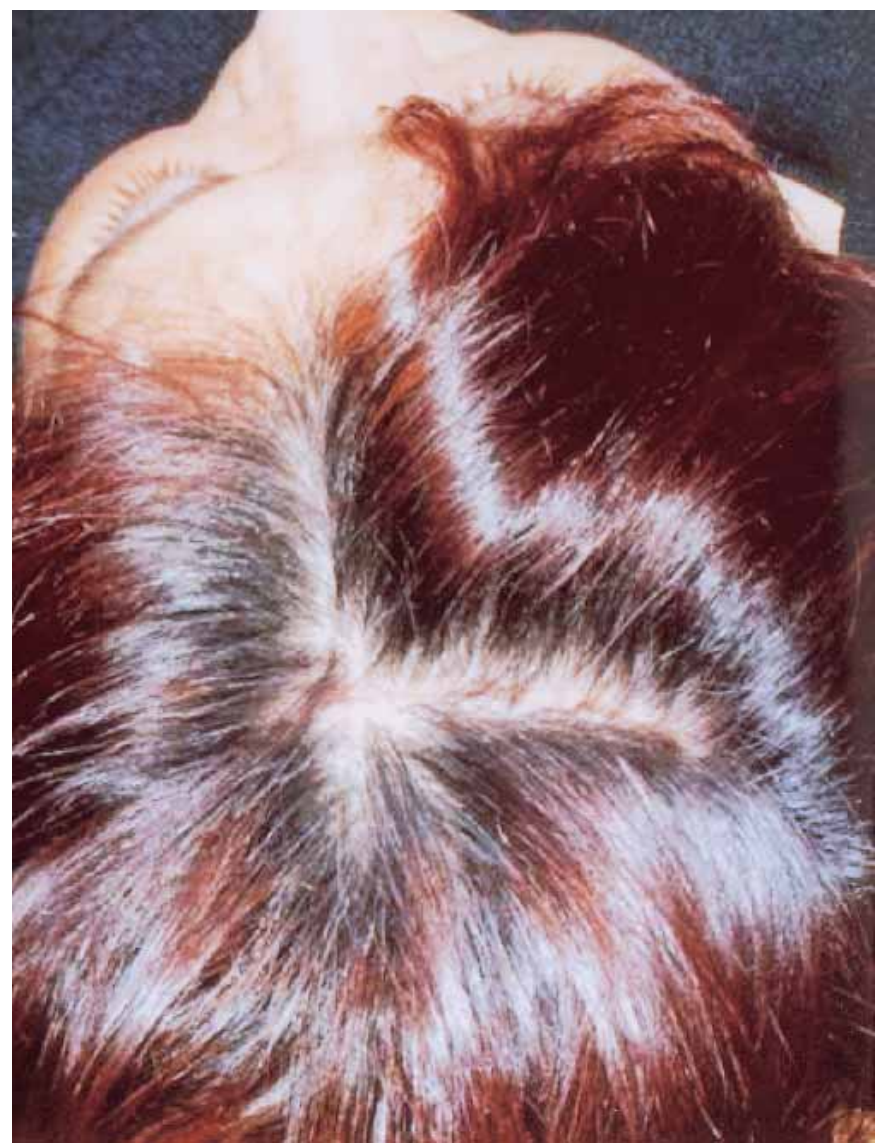

Figura 6 d: Postoperatorio a los 2 meses de evolución. 


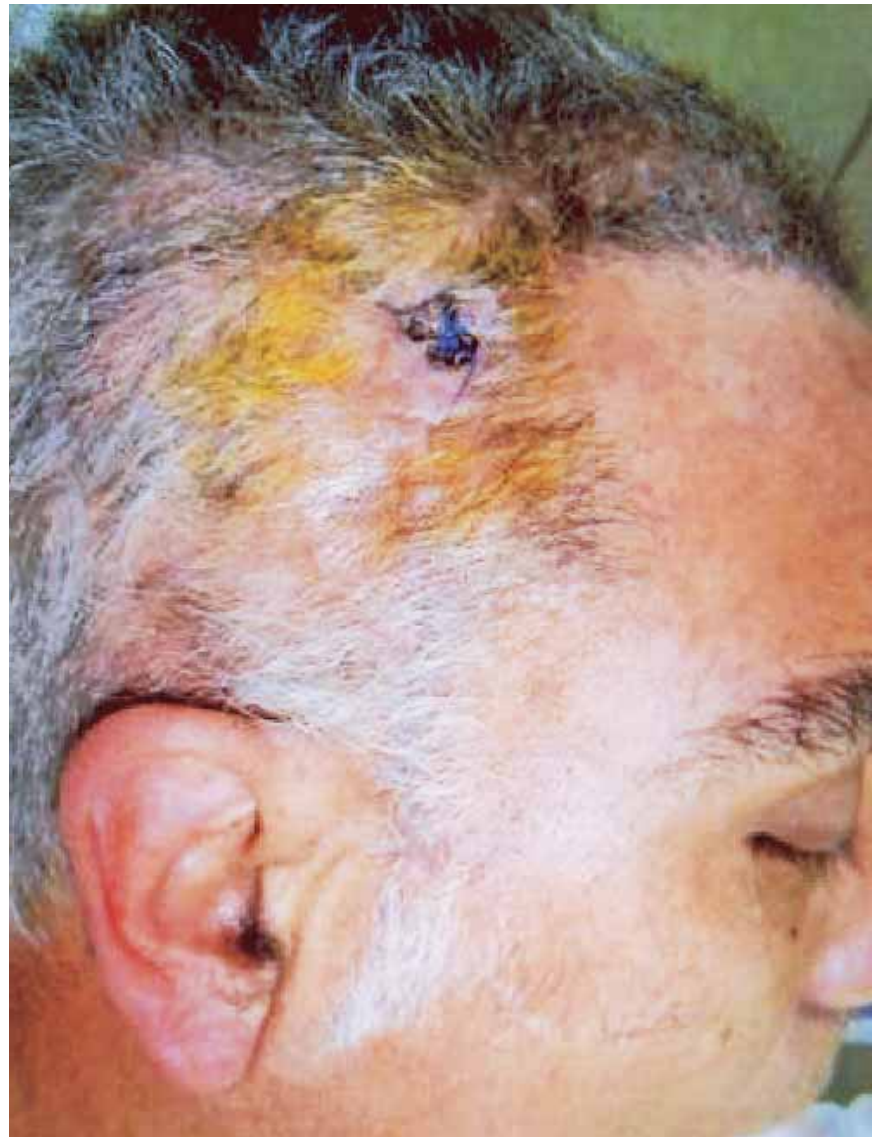

Figura 7 a: Varón de 62 años con exposición de malla de titanio temporal de $2 \mathrm{~cm}$. de diámetro secundaria a cirugía de aneurisma de arteria cerebral media y resutura.

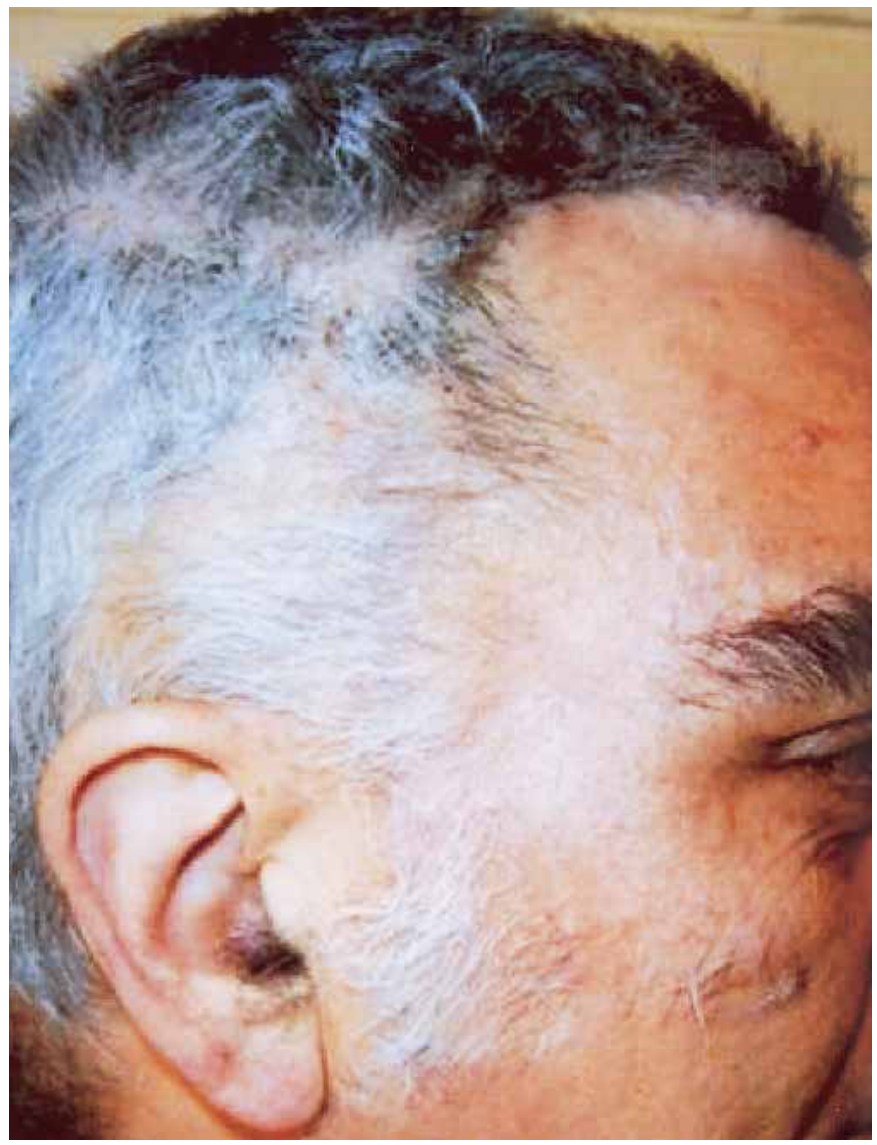

Figura 7 c: Postoperatorio a los 2 meses de evolución.

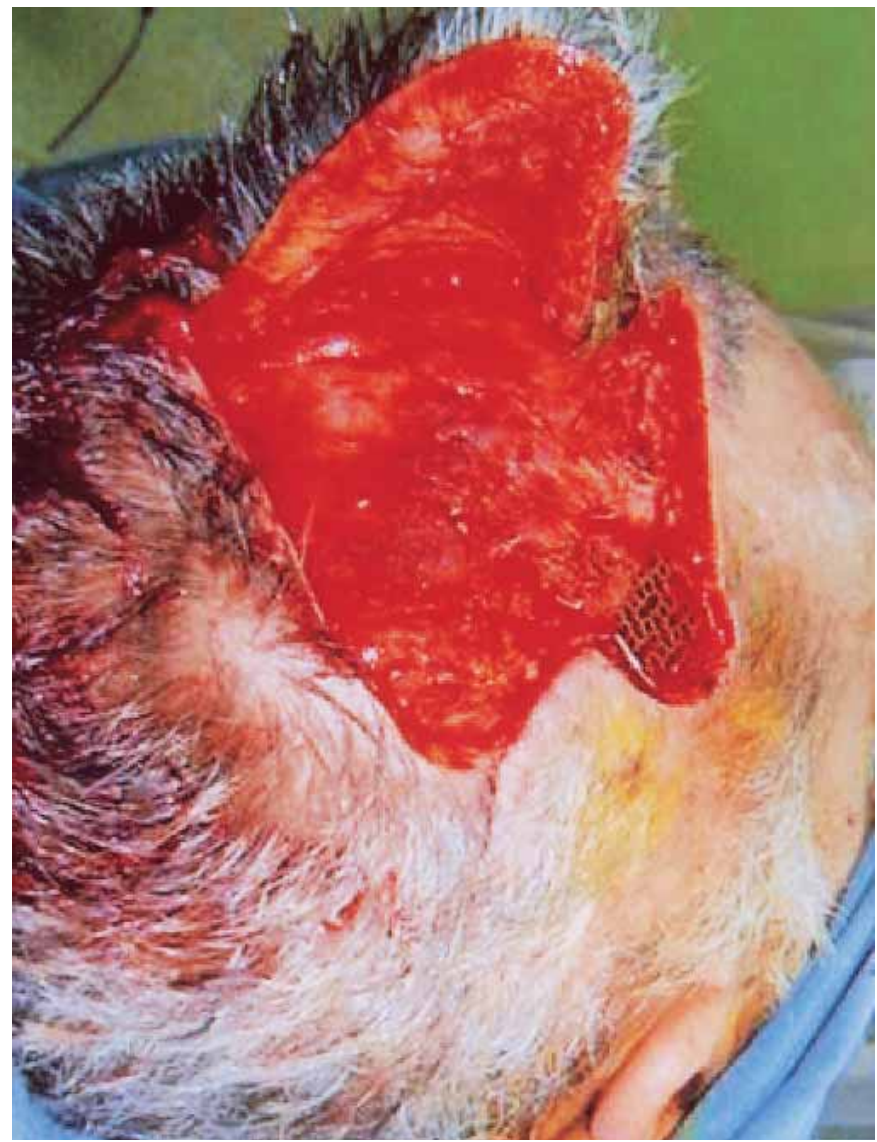

Figura 7 b: Resección de piel desvitalizada de $3 \mathrm{~cm}$. de diámetro y preparación de colgajo semilunar de base en arteria temporal superficial contralateral.

quen en la región retroauricular. Deben evitarse los colgajos coronales con base por delante de la oreja para ser transpuestos hacia atrás o viceversa, ya que se desplazan con mucha dificultad y exigen suturas a tensión. Empleamos este tipo de colgajo con preferencia en reconstrucciones secundarias a resecciones tumorales o alopecias.

Un elemento relevante para escoger el diseño del colgajo es la ubicación del defecto. El pedículo vascular del colgajo debe orientarse en el sentido del eje de alguna de las 12 arterias que irrigan el cuero cabelludo (2). Esta estrategia la hemos mantenido siempre consiguiendo buenos resultados.

\section{Conclusiones}

Una buena estrategia tradicional fue cerrar un defecto de $\mathrm{CC}$ con un injerto dermo epidérmico (17, 18). Debido a una constante labor de investigación, la Cirugía Plástica se encuentra en un permanente desplazamiento hacia nuevas fronteras; la tendencia actual es la reconstrucción inmediata, con la menor cantidad de procedimientos, a un costo razonable y utilizando técnicas basadas en evidencias extrarídas de la Cirugía Experimental y de la clínica (11- 15). Esta estrategia de reconstrucción es la que hemos 


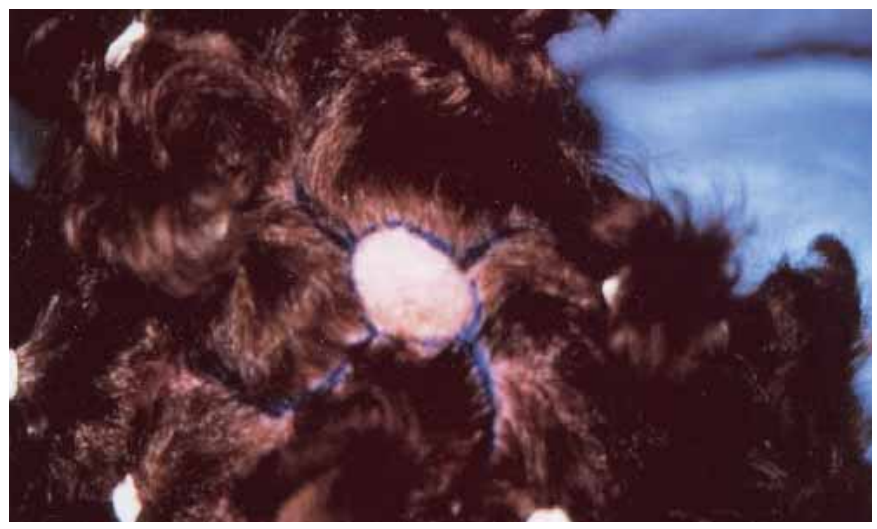

Figura 8 a: Alopecia occipito-parietal de $5 \mathrm{~cm}$. de diámetro.

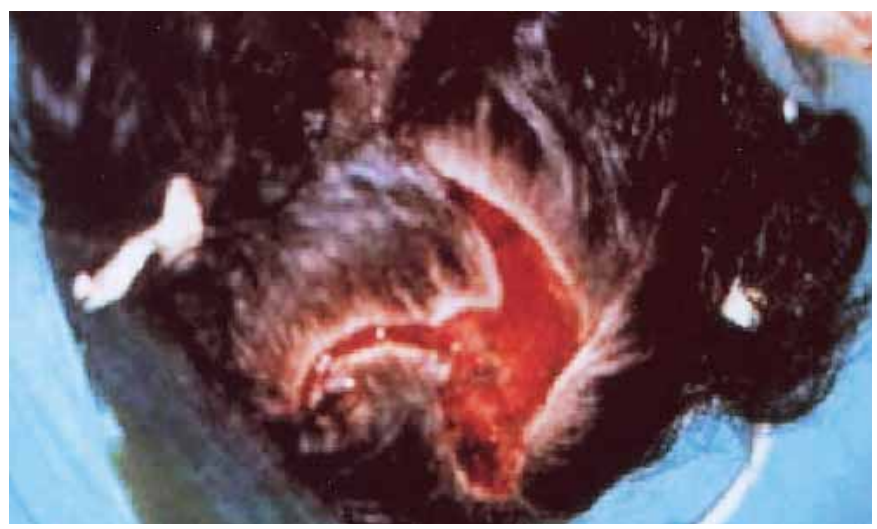

Figura 8 b: Resección y preparación de 4 colgajos circulares en remolino.

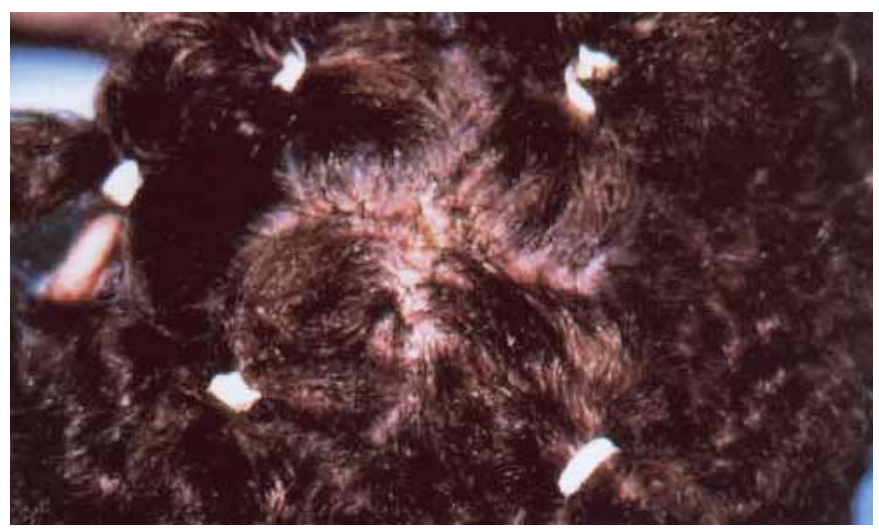

Figura 8 c: Postoperatorio a las 3 semanas seguido en nuestro protocolo y la recomendamos por su seguridad, sencillez y buenos resultados.

\section{Dirección del autor}

Dr. Osvaldo Iribarren Brown

Gabriela Mistral 2381, La Serena. Chile

e-mail: oiribarren@123.cl

\section{Bibliografía}

1. Mc Graw M: Scalping: "The savage and the surgeon". Clin Plast Surg 1983;10 (4): 679.

2. Reed O, Dingman R, Argenta L: "The surgical repair of traumatic defects of the scalp". Clin Plast Surg 1982; 9 (2): 131 .

3. Oishi S, Luce E: "The difficult scalp and skull wound". Clin Plast Surg 1995; $22(1): 51$.

4. Austad DE: "The origin of the expanded tissue". Clin Plast Surg 1987; 14: 431

5. wikipedia.orglwikilelipse

6. Vechione TR: "Multiple pinwheel scalp flaps". En Strauch B; Vasconez LO et al, 1990: Grabb's Encyclopaedia of flaps; Little Brown \& Co 1990 Pp $11-2$.

7. Emmet AJ, Catt PB, Atkinson L: "Cranial defects". En Barron J, Saad M: Operative Plastic \& Reconstructive Surgery. Churchill Livingstone, New York 1990 Pp $513-26$.

8. Pub Med: NCBI; National Library of Medicine, 2005. http://www.ncbi.nlm.nih.gov/entrez/query.

9. Mustoe T, Corral C: "Soft tissue reconstructive choices for craneal reconstruction". Clin Plast Surg 1995; 22: 543.

10. Iribarren O: "Reconstrucción de cuero cabelludo". Cuader Cir U.A.Ch 2000; 14 (1): 80.

11. Bishko Y, Slesarenko S: "Operative treatment of deep burns of the scalp and skull". Burns 1992; 18: 220.

12. Legbo JN, Shehu BB: "Managing scalp defects in sub-Saharan Africa". East Afr Med J. 2004 Feb; 81(2):87.

13. Iida N, Ohsumi N, Tonegawa M, Tsutsumi Y.: "Reconstruction of scalp defects using simple designed bilobed flap". Aesthetic Plast Surg 2000; 24 (2): 137.

14. Seline PC, Siegle RJ: "Scalp reconstruction". Dermatol Clin. 2005 Jan;23(1):13.

15. Newman MI, Hanasono MM, Disa JJ, Cordeiro PG, Mehrara BJ: "Scalp reconstruction: a 15-year experience". Ann Plast Surg. 2004;52(5):501.

16. Kroll S, Margolis R: "Scalp flap rotation with primary donor site closure". Ann Plast Surg 1993; 30: 452.

17. Alpert B, Buncke h, Mathes S: "Surgical treatment of the totally avulsed scalp". Clin Plast Surg 1992; 9: 145.

18. Bishko Y, Slesarenko S: "Operative treatment of deep burns of the scalp and skull”. Burns 1992; 18: 220. 